\title{
Benefits of forecasting and energy storage in isolated grids with large wind penetration - The case of Sao Vicente
}

\author{
Shengxi Yuan ${ }^{\mathrm{a},}{ }^{*}$, Ayse Selin Kocaman ${ }^{\mathrm{b}}$, Vijay Modi ${ }^{\mathrm{c}}$ \\ a Department of Mechanical Engineering, Columbia University, 160 Riverside Blvd, Apt 11T, New York, NY, 10069, USA \\ ${ }^{\mathrm{b}}$ Department of Industrial Engineering, Bilkent University, Endustri Muhendisligi, Ankara, Turkey \\ ${ }^{\mathrm{c}}$ Department of Mechanical Engineering, Columbia University, 500 W 120th St, Mudd 220, New York, NY, 10027, USA
}

\section{A R T I C L E I N F O}

\section{Article history:}

Received 22 December 2015

Received in revised form 9 December 2016

Accepted 22 December 2016

Available online 22 December 2016

\section{Keywords:}

Isolated grid

Wind speed forecasting

Rolling horizon

ARIMA model

Monte Carlo simulation

Storage

\begin{abstract}
A B S T R A C T
For electric grids that rely primarily on liquid fuel based power generation for energy provision, e.g. one or more diesel gensets, measures to allow a larger fraction of intermittent sources can pay-off since the displaced is high cost diesel powered generation. This paper presents a case study of Sao Vicente, located in Cape Verde where a particularly high fraction of wind capacity of 5.950 MW (75\% of the average demand) is installed, with diesel gensets forming the dispatchable source of power. This high penetration of intermittent power is managed through conservative forecasting and curtailments. Two potential approaches to reduce curtailments are examined in this paper: 1) an improved wind speed forecasting using a rolling horizon ARIMA model; and 2) energy storage. This case study shows that combining renewable energy forecasting and energy storage is a promising solution which enhances diesel fuel savings as well as enables the isolated grid to further increase the annual renewable energy penetration from the current $30.4 \%$ up to $38 \%$ while reducing grid unreliability. In general, since renewable energy forecasting ensures more accurate scheduling and energy storage absorbs scheduling error, this solution is applicable to any small size isolated power grid with large renewable energy penetration.
\end{abstract}

๑) 2016 Elsevier Ltd. All rights reserved.

\section{Introduction}

The lack of a large interconnected electrical grid fed with lowcost generation sources (such as large plants powered by coal, gas, nuclear or hydropower) in small island states has meant that power grids in such settings must rely on generators fueled by diesel. Historically, baseload, load following, and regulating units were all served by diesel generation which allowed generators faster ramp up compared with coal or nuclear power. These dieselbased isolated power grids have high operating costs due to the dependence on expensive fuel imports. To alleviate costs, these settings are increasingly installing a renewable source, typically wind [1]. While wind or solar can reduce operating costs, a high penetration of intermittent source has disruptive effects to system reliability and stability $[2,3]$. This paper focuses on the integration of wind power.

In order to mitigate the impacts of variability, various solutions

\footnotetext{
* Corresponding author.

E-mail addresses: sy2462@columbia.edu (S. Yuan), selin.kocaman@bilkent.edu. tr (A.S. Kocaman), modi@columbia.edu (V. Modi).
}

are potentially viable, such as geographical dispersion of wind turbines, demand-side management, wind curtailment, wind speed forecasting, and energy storage systems. Geographical dispersion of wind turbines tends to reduce the higher frequency fluctuations, thus reducing the need for control measures otherwise needed under more erratic wind power output regimes [4]. However, due to the limited terrestrial size of isolated grids, the effect of geographic dispersion is, of course, somewhat limited $[2,5]$. Demand-side management (DSM) shaves peak demands in the system but is in general more suitable when solar power is utilized instead of wind since solar is more predictable than wind due to its clear diurnal patterns [6]. Typically, solar generation combined with DSM is applied to air-conditioning loads [7]. Wind curtailment, enforced by the system operator, leads to lower wind turbine generator output compared to what could be otherwise produced given available wind resources. This is one of the essential tools to balance supply and demand as well as maintain voltage and frequency requirements particularly for small isolated grids. . However, curtailments strongly affect the revenue of wind farms. In other words, because wind farms have substantial capital cost but no fuel costs and minimum variable costs, dispatch below 
maximum output encumbers the system to recover its capital costs [8]. Lacking sophisticated smart controls and DSM, grid operators in small island states must resort to conservative forecasts of wind power and ask wind power units to limit their generation to prespecified thresholds, providing diesel units clear guidance on when and which ones need to be operating. The introduction of improved forecasts for wind can potentially lead to higher penetration of wind energy through improved dispatch and operating rules for plants that are load following plants or providing ramping duty $[2,9]$. Energy storage is another potential approach for smoothing variability and for recovering some of the losses due to curtailments [10-14]. Recent studies show that energy storage plays an essential role in power dispatching strategies in micro-grid systems [12-14].

This paper presents a case study for Sao Vicente. In Sao Vicente, a small island located in Cape Verde in the Atlantic Ocean, the electrical grid is an isolated grid with wind power integrated but still primarily relies on diesel fueled generation for baseload, load following, and regulation. Even though these type of grid are not uncommon, Sao Vicente is a particularly interesting case to study due to its high wind resource availability (as discussed in Section 2), enabling a large potential for deep penetration of renewable energy generation to reduce reliance on expensive fuel imports. Actual data from Sao Vicente in 2013 including hourly mean wind speeds, hourly wind energy potential generation, actual wind energy delivery, and demand data were obtained and used to study the overall energy situation. The paper aims at exploring the benefits of implementing a statistical wind speed forecasting model in conjunction of a potential energy storage system using real time series data.

\section{Background}

\subsection{Time series models for forecasting and simulation}

The majority of wind forecasting techniques can be clustered into two main groups, namely physical methods and statistical methods. The first group takes into account the meteorological considerations and utilizes the Numerical Weather Prediction (NWP) models which provide a weather forecast from the mathematical model of the atmosphere [9]. Commonly reported in literature, the NWP model outputs can be used directly or with other programs to estimate wind speeds [15-17]. These weather based models are able to provide reasonable predictions for longer horizon but require large computational resource and time. The second group aims at describing the relation between historical time series of wind speed at the location of interest [9]. Several time series based forecasting techniques have been proposed by researchers, including Autoregressive (AR) model by Huang and Chalabi [18], Autoregressive Moving Average (ARMA) model by Torres et al. and by Kamal and Jafri $[19,20]$, fractional-Autoregressive Integrated Moving Average (f-ARIMA) model by Kavasseri and Seetharaman [21], and the more general Autoregressive Integrated Moving Average (ARIMA) by Sfetsos [22]. Time series models require historical data but they are straightforward to implement and their short term (hours) prediction errors are relatively low. Other adoptions of time series-based wind forecasting, such as Fuzzy Logic (FL), Artificial Neural Networks (ANNs), Support Vector Machines (SVMs), Bayesian Networks (BNs) and Genetic Programming (GP), are also available but in general they require more effort due to high complexity [9].

Another advantage of time series models is that they provide confidence intervals for the forecasts, which enables the simulation of wind speed time series data. The use of time series models for simulating hourly mean wind speed time series is reported in literature commonly [23-25]. Due to the high dependence of hourly mean wind speed time series, using time series models for simulation incorporates the dependence structure of the observations [20]. Therefore, the simulated wind speed time series using real data have much higher fidelity compared to Weibull distribution random numbers simulation which does not incorporate the dependence structure and is usually used when real time series data is absent [26].

\subsection{Data - Sao Vicente}

In Sao Vicente, a wind/diesel isolated power grid has been implemented. In 2013, the electricity demand in Sao Vicente averaged $7961 \mathrm{kWh}$ per hour with lowest and highest demand being $5188 \mathrm{kWh}$ to $11,770 \mathrm{kWh}$ per hour respectively as shown in Fig. 1. The installed wind capacity in the system is $5950 \mathrm{~kW}$ (seven Vestas V52-850 kW turbines). The average wind speeds was at a staggering $8.8 \mathrm{~m} / \mathrm{s}$ and in the absence of curtailment the wind farm would have had a capacity factor of $51.9 \%$. The actual capacity factor with curtailment for 2013 was 40.7\%. Even with curtailment, the instantaneous power penetration reached above $80 \%$ and at one instance $100 \%$ as shown in Fig. 2 and the annual energy penetration rate reached $30.4 \%$.

Out of the total demand of 69.7 GWh in 2013, the annual wind energy dispatched was $21.2 \mathrm{GWh}$. While wind could have provided 27.1 GWh, due to curtailments only $21.2 \mathrm{GWh}$ was dispatched, and thus $5.9 \mathrm{GWh}$ is lost. In the current grid, the system operator manually determines wind power curtailment setpoints based on a rudimentary method, which is taking either the minimum or average of the last several hours observed. Setpoints are thresholds such that if a wind turbine produces power higher than a threshold value, the delivered power will be curtailed down to that value, and otherwise all power produced is delivered to the grid. The wind setpoints are usually conservative in order to avoid penalties. As shown in both Figs. 1 and 3, wind power is actively being curtailed to the level of outputs that are below potential given the resources available. In Fig. 3, the potential wind energy that could be produced at various wind speed is shown by the manufacturer's power curve. Also, some clear setpoint values are shown. Note that at low wind speeds, some actual energy delivered data are higher than the manufacturer's power curve because the wind speeds are hourly average and the energy delivered data are the sum over 7 turbines combined. During some hours where the mean wind speeds are
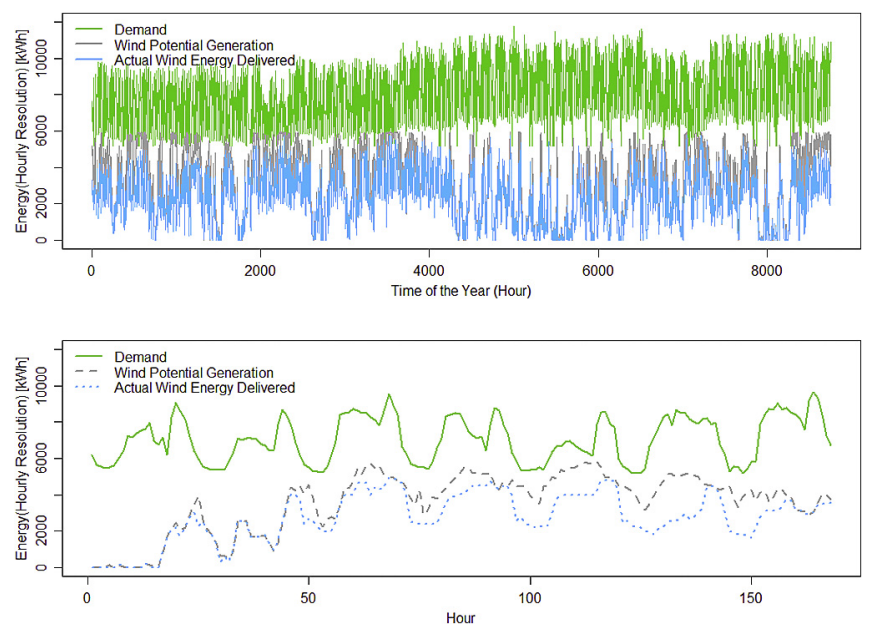

Fig. 1. Yearly Sao Vicente energy data in 2013 and a typical week (March 5th to Match 11 th). 


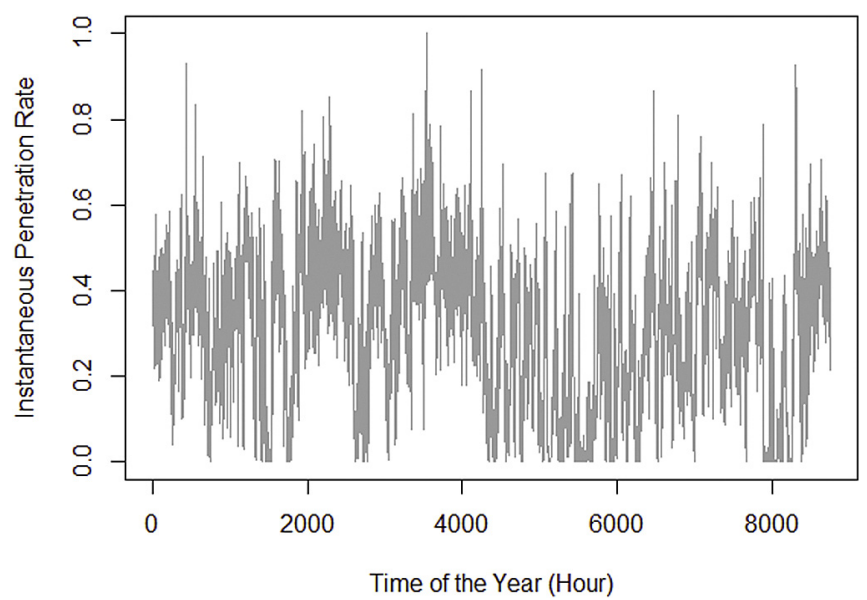

Fig. 2. Actual wind instantaneous penetration rate in 2013.

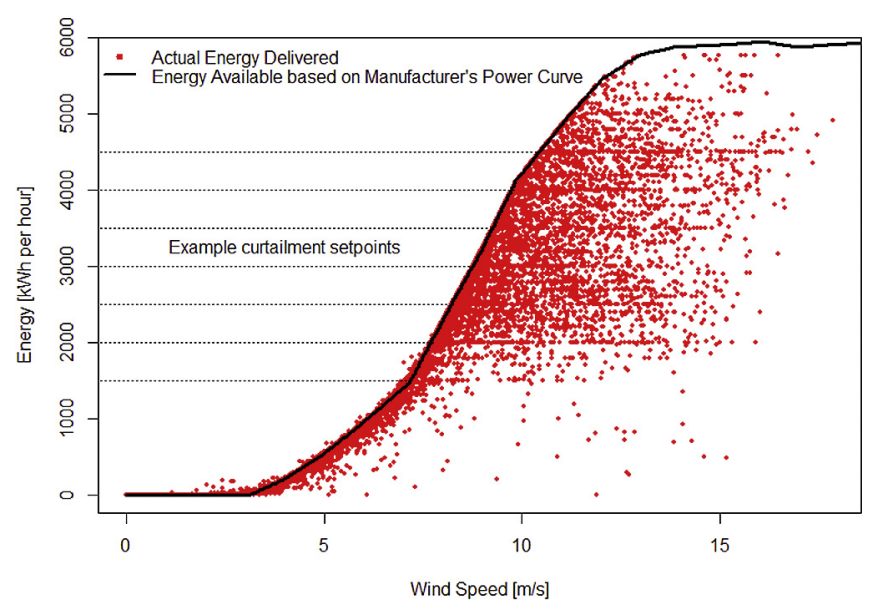

Fig. 3. Curtailed wind outputs.

low, the instantaneous wind speeds might be high and some turbines might have high production.

\section{Methodology}

One of the advantages of time series models is that the resource costs of implementation are low. Therefore, time series forecasting models can be easily applied based on a short term (one hour) rolling horizon mechanism. In other words, at every hour, a forecast for next hour is produced using historical time series as training data. In this paper, observed Sao Vicente wind speed time series data were trained on a rolling horizon basis using general ARIMA models and the model forecasts were tested for accuracy and compared with the persistence model. Based on the marginal probability distributions of the ARIMA model forecasts for each time horizon (each hour), 100 scenarios (yearly time series) of hourly mean wind speeds were simulated using the Monte Carlo method. Then, the simulated scenarios were used to evaluate various wind farm operating strategies, or curtailment setpoint rules, including setting setpoints based on ARIMA forecast, persistence method, and naïve methods which take the minimum or average of the last several hours. Furthermore, a potential storage system at various capacities was considered and the benefits of adding a storage system were evaluated.

\subsection{Wind speed forecasting}

The simplest and the mostly widely used method of wind speed forecasting is the persistence model which does not require any modelling. For a given time series $\{y(t)\}$, the persistence forecast is obtained by simply setting $\widehat{y}(t+1)=y(t)$, which implies that the average wind speed forecast for the next hour is equal to the average wind speed over the current hour. Both in literature and in practice, persistence model is the most frequently used benchmark method in wind speed forecasting since it is reasonable to assume that wind speeds are highly correlated in short terms [21].

In this paper, Autoregressive Integrated Moving Average or $\operatorname{ARIMA}(p, d, q)$ model is used for wind speed forecasting. This model, along with many self-adaptive or artificial intelligence techniques, can also be used in other applications including load forecasting [27]. ARIMA model, introduced by Box and Jenkins, is a versatile class of model for time series based forecasting, and has been applied to a wide variety applications [28]. The ARIMA forecasting equation for a stationary time series is a linear combination of the predictors that consist of lags of the observations and/or lags of the forecast errors. In time series forecasting, the goal is to predict a series that typically is not deterministic but rather contains a random component, such as wind speeds time series. If this random component is stationary, meaning that the statistical properties such as mean, variance and autocorrelation are all constant over time, then powerful techniques can be developed to forecast the future values of a series [29]. A time series that needs to be differenced to ensure stationarity is said to be an "Integrated" version of a series. The parameter $d$ in the $\operatorname{ARIMA}(p, d, q)$ denote the $d$ th difference of a time series $\{y(t)\}$.

$$
\begin{aligned}
& \text { If } d=0, Y_{t}=y_{t} \\
& \text { If } d=1, Y_{t}=y_{t}-y_{t-1} \\
& \text { If } d=2, Y_{t}=\left(y_{t}-y_{t-1}\right)-\left(y_{t-1}-y_{t-2}\right)
\end{aligned}
$$

Typically, a time series can achieve stationarity by differencing zero or one time $(d<2)$. Lags of the observations of the stationary series in the ARIMA forecasting equation are the "Autoregressive" terms. The parameter $p$ refer to the order of the Autoregressive process or the number of autoregressive terms used as predictors. Lags of the forecast errors of the stationary series in the ARIMA forecasting equation are the "Moving Average" terms. The parameter $q$ refer to the order of the Moving Average process or the number of moving average terms used as predictors [29].

Let $\{y(t)\}$ represent the time series of hourly mean wind speeds in Sao Vicente. Then, an $\operatorname{ARIMA}(p, d, q)$ formulation for the time series can be described by,

$\varphi(B)(1-B)^{d} y_{t}=\theta(B) Z_{t}$

where $Z t$ is white noise $\left(\sim W N\left(0, \sigma^{2}\right)\right)$, and $B$ is the backshift operator defined by $B\left(y_{t}\right)=y_{t-1}$. The functions $\phi$ and $\theta$ are polynomial functions of the backshift operator $B$ given by,

$\varphi(B)=1-\varphi_{1} B-\varphi_{2} B^{2}-\ldots-\varphi_{p} B^{p}$

$\theta(B)=1+\theta_{1} B+\theta_{2} B^{2}+\ldots+\theta_{q} B^{q}$

Given an hourly wind speed time series $\{y(t)\}$, an ARIMA model fit can be selected based on the AIC(Akaike information criterion) statistic. The AIC statistic is defined as: 
$\operatorname{AIC}(\varphi, \theta)=-2 \ln L\left(\varphi, \theta, \frac{S(\varphi, \theta)}{n}\right)+\frac{2(p+q+1) n}{(n-p-q-2)}$

where $L$ is the likelihood of the data under the Gaussian ARMA model with parameters $\left(\varphi, \theta, \sigma^{2}\right), n$ is the sample size, and $S(\varphi, \theta)$ is the residual sum of squares. The parameters $(p, q)$ and the functions $(\varphi, \theta)$ in an ARIMA model are chosen by minimizing the AIC. This can be achieved using the "Forecasting: principles and practice" package in R, which is a widely used open source statistical software [30]. In addition, the next hour forecast, $\widehat{y}(t+1)$, can be outputted using the ARIMA forecast equation calculated by the "auto.arima" function in the R package.

For ARIMA forecasting, the forecasting accuracy declines as the forecasting time horizon increases. In this paper, hourly rolling time horizon forecasting technique is used in order to obtain the highest forecasting accuracy. For each hour, an ARIMA model was autofitted using the historical wind speed time series as training data and the forecasted wind speed for that hour is the expected next hour forecast of the corresponding ARIMA model. For example, if the size of training data is $\mathrm{n}$ hours and the wind speed to be forecasted at hour $\mathrm{t}$ is $\widehat{y}(t)$, then a specific $\operatorname{ARIMA}(p, d, q)$ model is autofitted to the historical time series $\{y(t-n), y(t-n+1), \ldots, y(t-2), y(t-1)\}$ and the forecasted wind speed at hour $t, \widehat{y}(t)$, is outputted by this $\operatorname{ARIMA}(p, d, q)$ model. Then, for the next hour, $\mathrm{t}+1$, another specific $\operatorname{ARIMA}(p, d, q)$ model is auto-fitted to the historical time series $\{y(t-$ $n+1), y(t-n+2), \ldots, y(t-1), y(t)\}$ and the forecasted wind speed at hour $t+1, \widehat{y}(t+1)$, is outputted by this new $\operatorname{ARIMA}(p, d, q)$ model. In addition, the confidence interval of the next hour forecast is outputted. Also, the error term follows a Normal distribution with mean zero and variance outputted as well.

The forecasting accuracy is measured using absolute percentage error, or APE. APE is defined by,

$A P E=|\widehat{y}(t)-y(t)| /|y(t)|$

where $\widehat{y}(t)$ is the forecasted wind speed at hour $t$ and $y(t)$ is the observed wind speed at hour $\mathrm{t}$.

The relationship between the size of training data (number of hours) and the forecasting APE for each month is shown in Fig. 4. The APE of the persistence model forecasting is shown in Fig. 5.

As shown in Figs. 4 and 5, in both the rolling horizon ARIMA
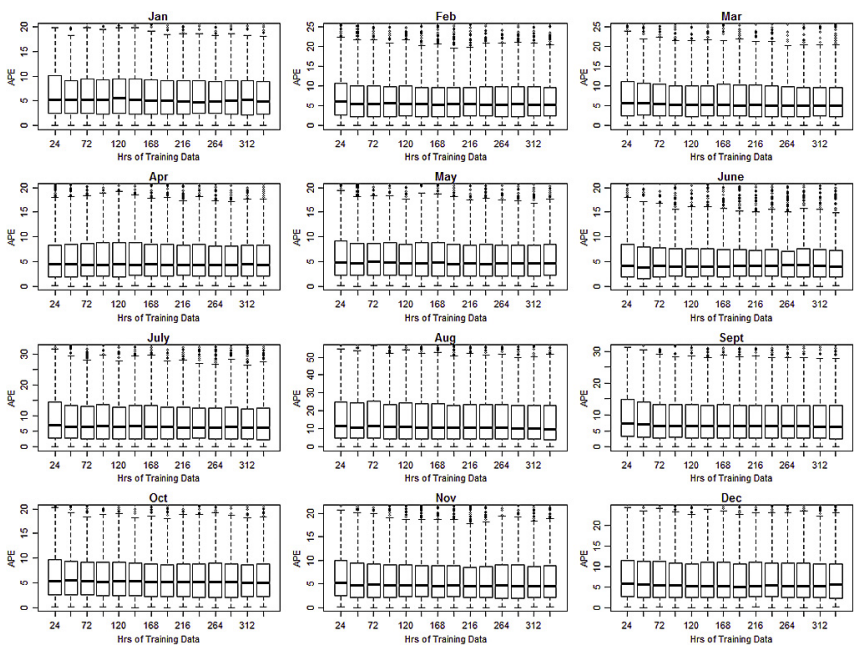

Fig. 4. Boxplots of wind speed forecasting APE at various training data sizes for each month suing rolling horizon ARIMA model.

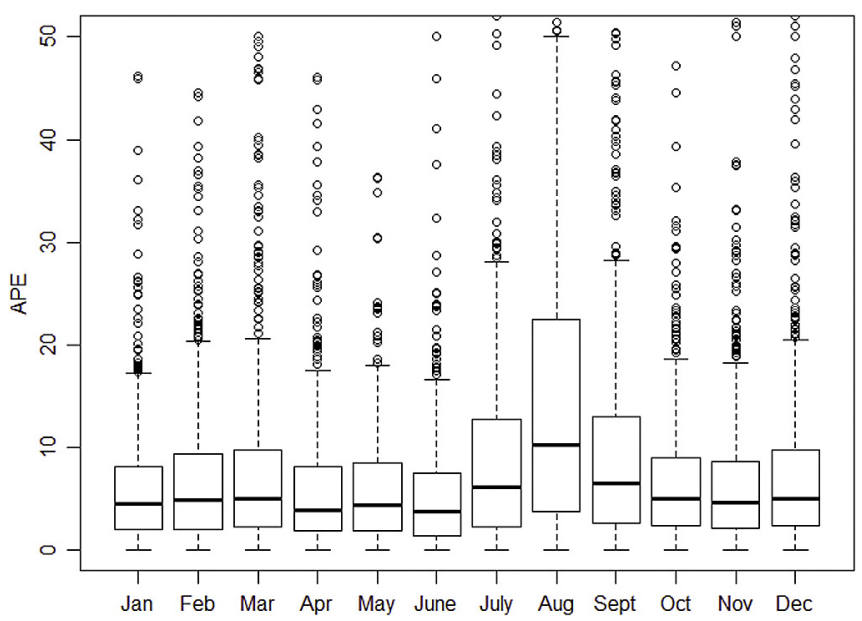

Fig. 5. Boxplots of wind speed forecasting APE for each month using Persistence model.

model and the Persistence model, the forecasting error is the highest in August because a large number of low or zero wind speeds were observed. For the rest of the year, on average the forecasting performance of the two models are comparable with APE roughly around $6 \%$. As expected, the persistence model produced a lot of large forecasting errors because the model does not require any modeling and does not consider any historical data expect for the previous time step. However, even though rolling horizon ARIMA models do take into account historical data, large forecasting errors are still present. Thus, it is not possible to conclude which forecasting method would produce better results in terms of lower curtailments and higher fuel savings. In fact, we must examine these forecasting methods under various combinations of operating strategies and storage capacities. On the other hand, Fig. 4 shows the effect of training data size on the forecasting accuracy of rolling horizon ARIMA models. Overall, it can be concluded by observing the median and the interquartile range that the forecasting accuracy as well as the forecasting consistency slightly improve as the size of the training data increases. However, the improvements in accuracy and consistency diminish as the size of the training data gets larger.

\subsection{Simulation}

\subsubsection{Wind speed and power}

As mentioned previously, the error term of next hour ARIMA forecast follows a Normal distribution with mean zero and variance known, enabling the Monte Carlo simulation. The version of Monte Carlo simulation used in the case study is introduced by Kamal and Jafri, which states that a simulated value of wind speed can be regarded as a one hour forecast to which a random error has been added [20]. Thus, the simulated mean wind speed in a given hour is the one hour forecast plus a random number that is generated from the marginal Normal distribution associated with error term of next hour ARIMA forecast for that hour. This can be described by,

$\tilde{y}(t+1)=\widehat{y}(t+1)+\varepsilon$

where $\tilde{y}(t+1)$ is the simulated wind speed at hour $t+1, \widehat{y}(t+1)$ is the ARIMA forecasted wind speed at hour $t+1$, and $\varepsilon$ is a normal random number generated from the Normal distribution associated with the ARIMA model for the hour $t+1$. Without overfitting and loss of generality while keeping reasonable forecasting accuracy and low computation time, each wind speed data simulated are 

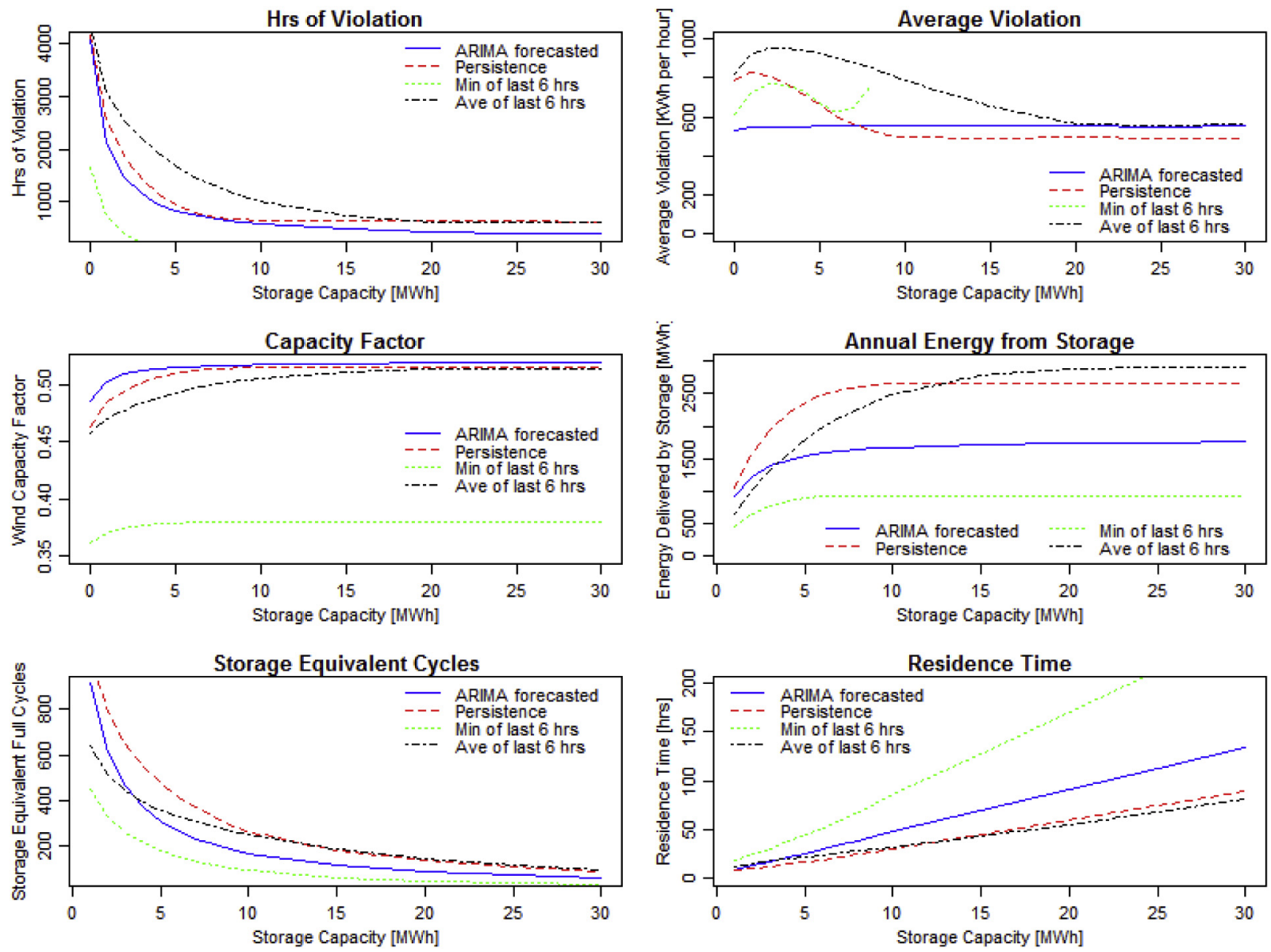

Fig. 6. Simulation results: expectations of evaluation metrics for four wind operating strategies for setpoints.

based on its specific ARIMA model with $72 \mathrm{~h}$ as training data size. Using the fitted ARIMA models described in Section 3.1, an hourly mean wind speed time series can be simulated for an entire year. Then, this process was repeated and 100 yearly time series of hourly mean wind speed data were generated and inputted as scenarios in the simulation. Using this method, the main statistical characteristics of the wind speed time series, include mean, variance, probability distribution, seasonality and autocorrelation functions, are reserved. Finally, the corresponding wind power time series were obtain using the manufacturer's turbine power curve.

\subsubsection{Operating strategies and energy storage}

Under the simulated scenarios of 100 yearly time series of hourly mean wind speeds, the following four different wind operating strategies were studied: the next hour wind curtailment setpoint is set 1 ) at the forecasted value using ARIMA model; 2) at the value of the previous hour using persistence model; 3 ) at the minimum of the last six hours observed; 4 ) at the average of the last six hours observed. An energy storage system with capacity ranging from $0 \mathrm{MWh}$ to $30 \mathrm{MWh}$ was also considered. The storage capacity was simulated up to only $30 \mathrm{MWh}$ since the size of the grid is small and large storage capacity is not sensible. Both discharging and charging efficiencies for the storage are assumed to be $90 \%$, however storage ramping and depth of discharge constraints are not considered. The demand time series for all scenarios is the real demand data from 2013 and is assumed to be the same for all scenarios.

\section{Results}

For each operating strategy and each storage capacity, the expected values of penalties, wind capacity factor, energy delivered by storage (fuel savings), storage equivalent cycles, and storage residence time were investigated as evaluation metrics. In Fig. 6, these expected values of evaluation metrics are plotted against storage capacity. Hours of violation is defined as the number of hours in a year where the actual wind and storage energy delivery is not able to meet the commitment level. In other words, the

Table 1

Expectations of results for no energy storage.

\begin{tabular}{|c|c|c|c|c|}
\hline & ARIMA forecasted & Persistence & Min of last six hours & Ave of last six hours \\
\hline Hours of violation & 4079 & 4150 & 1662 & 4331 \\
\hline Average violation [kWh] & 532 & 788 & 617 & 822 \\
\hline Wind capacity factor & $48.5 \%$ & $46.3 \%$ & $36.1 \%$ & $45.8 \%$ \\
\hline
\end{tabular}


curtailment setpoints are higher than the actual wind energy and the energy in storage combined. In this case, fast-ramping back-up diesel generation kicks in to meet the overall demand and the average amount needed is represented by the average violation. Hence, hours of violation and average violation represent the penalty when back-up diesel is required. Capacity factor represents the wind energy utilization. For isolated grids which heavily rely on fuel imports, high capacity factor is desirable for economic considerations. Annual energy from storage, storage equivalent cycles and residence time are metrics to describe the storage utilization rate, which is a crucial criterion for the implementation of storage. The no storage case, which is the benchmark case in this study, is summarized in Table 1 . The diesel fuel requirements and total savings are shown in Fig. 7.

\subsection{Discussion}

In this section, the simulation results of the storage case with both wind forecasting and energy storage considered are discussed and compared against the no storage case where only wind forecasting is considered.

\subsubsection{No storage case}

The persistence model is competitive with the ARIMA model in terms of the forecasting APE shown in Figs. 4 and 5. This result is also reflected in the no storage case in Table 1 where ARIMA forecasting does not provide significant advantage over persistence forecasting. ARIMA forecasting barely prevails in the wind capacity factor category and the system penalty is still significant in terms of the number of violating hours. However, due to lower variance in forecasting error as mentioned previously, the average violation is lower for ARIMA forecasting. The most conservative strategy of the four is setting the next hour setpoint as the minimum of the last six hours observed, which reduces penalty greatly but sacrifices in term of capacity factor. Setting setpoint as the average of the last six hours observed is the most risky strategy because the probability of violation is the highest.

\subsubsection{Storage case}

When energy storage system is present, the advantages of ARIMA forecasting become more apparent. As seen in Fig. 6, the hours of violations rapidly drop as storage capacity increases for all four operating strategies, with ARIMA forecasting much safer than the persistence forecasting at low storage capacities (2-5 MWh). Furthermore, with ARIMA forecasting at low storage capacities, the wind capacity factor is $3-4 \%$ higher than persistence model and the average violation is much lower. Also, the wind capacity factor reaches $50 \%$, which is $10 \%$ higher than the current situation, and the annual energy penetration rate can be calculated to have an increase from the current $30.4 \%-38 \%$. On the other hand, ARIMA forecasting also shows promising results in terms of fuel savings in Fig. 7. When storage is initially implemented, small violations are absorbed thus the average violation slightly increase. As more storage capacity is implemented, the average violations converge to a constant level for all four strategies. For ARIMA, persistence, and average of the last six hours strategies, both hours of violation and average violations converge to a non-zero level, meaning that the storage always fail to absorb the extreme cases where the next hour wind is dramatically different than the setpoints. For the minimum of the last six hours strategy, it is seen that at $8 \mathrm{MWh}$ storage capacity the violations are only the extreme cases and at $9 \mathrm{MWh}$ capacity all violations are prevented, which gain confirms that the minimum of the last six hours rule is the most conservative strategy.
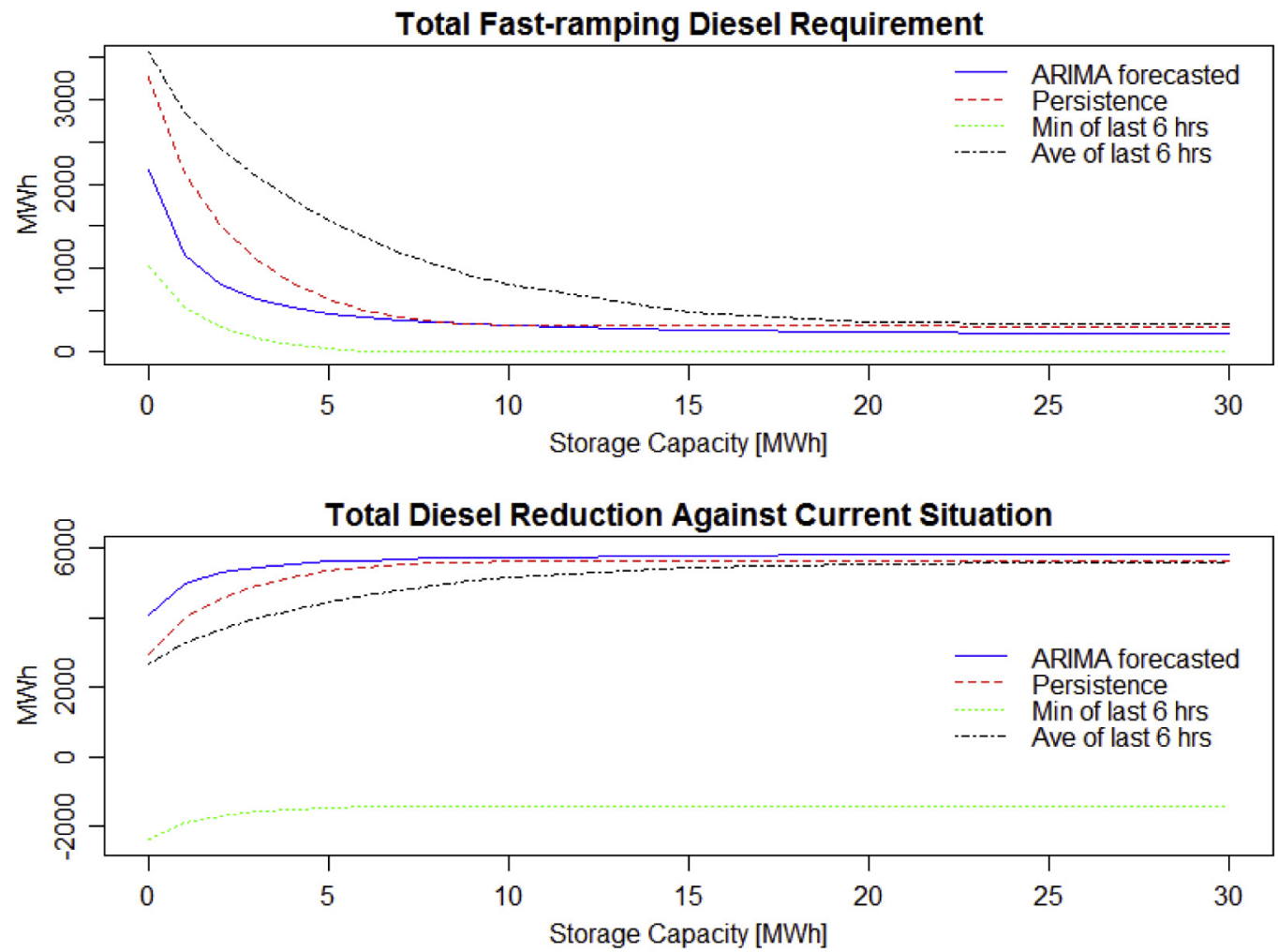

Fig. 7. Fast-ramping diesel requirements and overall system fuel savings. 


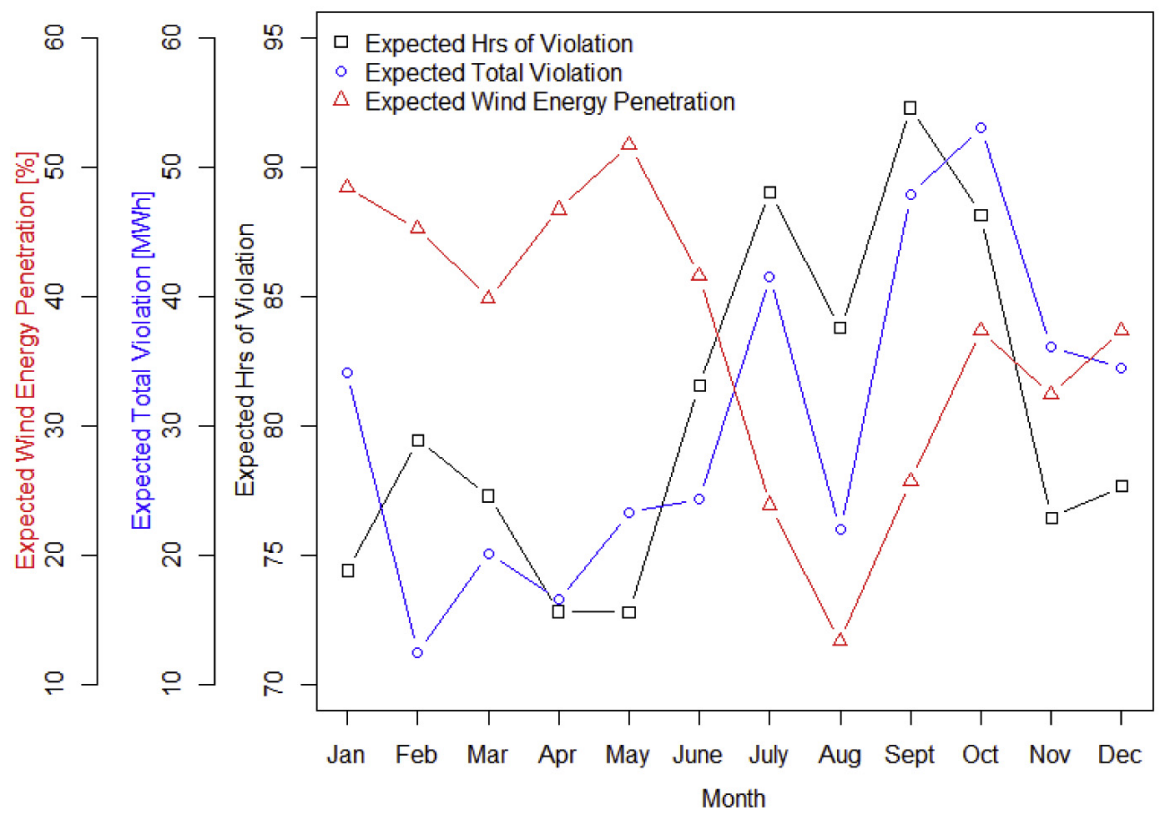

Fig. 8. Expected hours of violation, total violation in MWh, and wind energy penetration by month for ARIMA forecasting and 5 MWh storage.

The benefit of storage diminishes as capacity increases. In Fig. 6, hours of violation, wind capacity factor, and annual energy plateau while the utilization rate continues to dip. In addition, fast-ramping diesel requirements and fuels savings cease to improve at large storage capacities as shown in Fig. 7. Therefore, from a purely energy point of view, implementing a small size energy storage system combined with rolling horizon ARIMA forecasting mitigates issues due to large wind penetration by reducing the number of instances where fast-ramping diesel is required and improves the economics of the system through diesel fuel savings.

In essence, ARIMA forecasting enables more accurate renewable energy scheduling and coupling ARIMA forecasting and energy storage absorbs the variability of renewable energy. Therefore, ARIMA forecasting together with energy storage is a promising solution for issues in isolated grids with large renewable penetration.

A specific example of implementing ARIMA forecasting and 5 MWh energy storage is studied and the expected hours of violation, total violation in MWh (fast ramping diesel requirements), and wind energy penetration by month are shown in Fig. 8. This particular combination of ARIMA forecasting and energy storage is interesting to study. $5 \mathrm{MWh}$ for storage is a reasonable size to invest for roughly 1 million dollars nowadays. Combining with forecasting, it enables a wind capacity factor of $51 \%$ and only 550 MWh of fast ramping diesel requirements annually. Out of the 550 MWh fast ramping diesel generation, the majority of it comes from July, September, and October when the hours of violations are relatively high and the wind energy penetrations are relatively low. In August when the wind speeds are the lowest, the wind penetration is the lowest and the total violation (fast ramping diesel) is also low. In the months where wind energy is abundant, i.e. February to June, wind penetrations are high and violations are low.

\section{Conclusion}

In this paper, a case study for Sao Vicente was performed to investigate potential solutions to mitigate issues in isolated power grids caused by large wind penetration. One of the promising solutions is time series based forecasting, which requires minor time and resource to implement and provides high forecasting accuracy. The general Autoregressive Integrated Moving Average model was used in forecasting wind speeds and it out-performs the benchmark persistence model. In the simulation study, it is shown that if no storage is present, implementing ARIMA forecasting alone does not significantly improves grid reliability and fuel saving is barely achieved. However, coupling ARIMA forecasting and energy storage together demonstrates significant improvements in grid reliability, wind capacity factor, and fuel saving. Large storage capacity is not desired since the benefit of storage diminishes and the economics becomes unjustifiable. Type of storage technology to implement and other options such as implementing additional wind generation or solar generation to further improve the grid are to be studied in future research.

\section{Acknowledgement}

The authors would like to thank Mr. Edmar Coronel from CABEOLICA, SA and Mr. Antão Cruz from ELECTRA, SARL for providing the data used in the analysis for this paper. The authors would also like to thank Prof. Antunio Barbosa from University of Cape Verde for the coordination.

\section{References}

[1] A. Malheiro, P. Castro, R. Lima, A. Estanqueiro, Integrated sizing and scheduling of wind/PV/diesel/battery isolated systems, Renew. Energy 83 (2015) 646-657.

[2] D. Weisser, R. Garcia, Instantaneous wind energy penetration in isolated electricity grids concepts and review, Renew. Energy 30 (2005) 1299-1308.

[3] M. Ahlstrom, R. Zavadil, The role of wind forecasting in grid operations \& reliability, in: IEEE/PES Transmission and Distribution Conference \& Exhibition, 2005.

[4] J. Apt, The spectrum of power from wind turbines, J. Power Sources 169 (2007) 369-374.

[5] Y. Luo, L. Shi, G. Tu, Optimal sizing and control strategy of isolated grid with wind power and energy storage system, Energy Convers. Manag. 80 (2014) 407-415.

[6] A. Al-Alawi, S.M. Islam, Demand side management for remote area power supply systems incorporating solar irradiance model, Renew. Energy 29 (2004) 2027-2036. 
[7] B. Bailey, J.R. Doty, R. Perez, R. Stewart, Evaluation of a demand side management photovoltaic system, IEEE Trans. Energy Convers. 8 (No. 4) (1993).

[8] L. Bird, J. Cochran, X. Wang, Wind and solar energy curtailment experience and practices in the United States, NREL Tech. Rep. (2014).

[9] A. Tascikaraoglu, M. Uzunoglu, A review of combined approaches for prediction of short-term wind speed and power, Renew. Sustain. Energy Rev. 34 (2014) 243-254

[10] A.S. Kocaman, C. Abad, T.J. Troy, W.T. Huh, V. Modi, A stochastic model for a macroscale hybrid renewable energy system, Renew. Sustain. Energy Rev. 54 (2016) 688-703.

[11] A.S. Kocaman, Essays on Infrastructure Design and Planning for Clean Energy Systems, Doctoral dissertation, Columbia University, 2015.

[12] R. Rigo-Mariani, B. Sareni, X. Roboam, C. Turpin, Optimal power dispatching strategies in smart-microgrids with storage, Renew. Sustain. Energy Rev. 40 (2014) 649-658.

[13] S. Mohammadi, S. Soleymani, B. Mozafari, Scenario-based stochastic operation management of MicroGrid including wind, photovoltaic, micro-turbine, fuel cell and energy storage devices, Electr. Power Energy Syst. 54 (2014) $525-535$.

[14] V.N. Coelho, I.M. Coelho, B.N. Coelho, M.W. Cohen, A.J.R. Reis, S.M. Silva, M.J.F. Souza, P.J. Fleming, F.G. Guimaraes, Multi-objective energy storage power dispatching using plug-in vehicles in a smart-microgrid, Renew. Energy 89 (2016) 730-742.

[15] L. Landberg, Short-term prediction of the power production from wind farms, J. Wind Eng. Ind. Aerodyna. 80 (1999) 207-220.

[16] M.S. Roulston, D.T. Kaplan, J. Hardenberg, L.A. Smith, Using medium-range weather forecasts to improve the value of wind energy production, Renew. Energy 28 (2003) 585-602.

[17] L. Lazic, G. Pejanovic, M. Zivkovic, Wind forecasts for wind power generation using the Eta model, Renew. Energy 35 (2010) 1236-1243.

[18] Z. Huang, Z.S. Chalabi, Use of time-series analysis to model and forecast wind speed, J. Wind Eng. Ind. Aerodyna. 56 (1995) 311-322.

[19] J.L. Torres, A. Garcia, M. De Blas, A. De Francisco, Forecast of hourly average wind speed with ARMA models in Navarre (Spain), Sol. Energy 79 (2005) $65-77$.

[20] L. Kamal, Y.Z. Jafri, Time series models to simulate and forecast hourly averaged wind speed in Quetta, Pakistan, Sol. Energy 61 (1997) 23-32.

[21] R.G. Kavasseri, K. Seetharaman, Day-ahead wind speed forecasting using fARIMA models, Renew. Energy 34 (2009) 1388-1393.

[22] A. Sfetsos, A novel approach for the forecasting of mean hourly wind speed time series, Renew. Energy 27 (2002) 163-174.

[23] H. Aksoy, Z.F. Toprak, A. Aytek, N.E. Unal, Stochastic generation of hourly mean wind speed data, Renew. Energy 29 (2004) 2111-2131.

[24] A. Sfetsos, A comparison of various forecasting techniques applied to mean hourly wind speed time series, Renew. Energy 21 (2000) 23-25.

[25] R. Billinton, H. Chen, R. Ghajar, Time-series models for reliability evaluation of power systems including wind energy, Microelectron. Reliab. 36 (1996) $1253-1261$.

[26] C. Gavriluta, S. Spataru, I. Mosincat, C. Citro, I. Candela, P. Rodriguez, Complete methodology on generating realistic wind speed profiles based on measurements, in: International Conference on Renewable Energies and Power Quality, 2012.

[27] V.N. Coelho, I.M. Coelho, B.N. Coelho, A.J.R. Reis, R. Enayatifar, M.J.F. Souza, F.G. Guimaraes, A self-adaptive evolutionary fuzzy model for load forecasting problems on smart grid environment, Appl. Energy 169 (2016) 567-584.

[28] G.E.P. Box, G.M. Jenkins, Time Series Analysis: Forecasting and Control, Revised ed., Holden-Day, San Francisco, 1976.

[29] P.J. Brockwell, Introduction to Time Series Forecasting, second ed., Springer, New York, 2002

[30] R [Computer software], 2015. Retrieved from, https://cran.r-project.org/bin/ windows/base/. 